\title{
Carotid intima-media thickness (IMT) in patients with severe familial and non-familial hypercholesterolemia: The effect of measurement site on the IMT correlation with traditional cardiovascular risk factors and calcium scores
}

\author{
Rafał Gałąska ${ }^{1}$, Dorota Kulawiak-Gałąska ${ }^{2}$, Magdalena Chmara ${ }^{3}$, Krzysztof Chlebus ${ }^{1}$, \\ Agnieszka Mickiewicz ${ }^{1}$, Andrzej Rynkiewicz ${ }^{4}$, Bartosz Wasąg ${ }^{3}$, Michał Studniarek², \\ Marcin Fijałkowski ${ }^{1}$, Marcin Gruchała ${ }^{1}$ \\ ${ }^{1} 1^{\text {st }}$ Department of Cardiology, Medical University of Gdansk, Poland \\ ${ }^{2}$ Department of Radiology, Medical University of Gdansk, Poland \\ ${ }^{3}$ Department of Biology and Genetics, Medical University of Gdansk, Poland \\ ${ }^{4}$ Department of Cardiology and Cardiosurgery, University of Warmia and Mazury, Olsztyn, Poland
}

\begin{abstract}
Background: The carotid intima-media thickness (IMT) measurement may be carried out proximally (DIMT) or distally (dIMT) in relation to the bulb of the common carotid artery which has significant implications on the results and correlation with risk factors. The aim of the study was to compare the pIMT and dIMT in patients with familial hypercholesterolemia confirmed by genetic testing (FH group) and patients with severe non-familial hypercholesterolemia, with negative results of genetic testing (NFH group) and to determine the correlation of results with traditional atherosclerotic risk factors and calcium scores.

Methods: A total of 86 FH and 50 NFH patients underwent pIMT and dIMT measurements of both carotid arteries as well as computed tomography (CT) with coronary and thoracic aorta calcium scoring. Results: The meanpIMT of both right and left common carotid artery were significantly higher in patients with FH compared to the NFH group (meanpRIMT $0.721 \pm 0.152$ vs. $0.644 \pm 0.156$, $p<0.01$, meanpLIMT $0.758 \pm 0.173$ vs. $0.670 \pm 0.110, p<0.01)$. Patient age, pre-treatment lowdensity lipoprotein (LDL) cholesterol levels (LDLmax) at baseline and systolic blood pressure were independent predictors of PIMT increases in both carotid arteries. Smoking history, age and LDLmax were independent predictors of dIMT increase. There was a significant correlation between the calcium scores of the ascending aorta, coronary artery and aortic valve and all IMT parameters.

Conclusions: The IMT measured proximally better between patients with familial and non-familial hypercholesterolemia. The association between IMT and traditional cardiovascular risk factors varies between measurement sites. IMT values correlate CT calcium scores in all patients with hypercholesterolaemia regardless of genetic etiology. (Cardiol J 2021; 28, 2: 271-278)
\end{abstract}

Key words: atherosclerosis, familial hypercholesterolemia, intima-media thickness, calcium scores, multidetector computed tomography

Address for correspondence: Dr. Rafał Gałąska, $1^{\text {st }}$ Department of Cardiology, Medical University of Gdansk, ul. Dębinki 7, 80-952 Gdańsk, Poland, tel: +48 58 3492500, fax: +48 58 3492504, e-mail: rgal@gumed.edu.pl

This article is available in open access under Creative Common Attribution-Non-Commercial-No Derivatives 4.0 International (CC BY-NC-ND 4.0) license, allowing to download articles and share them with others as long as they credit the authors and the publisher, but without permission to change them in any way or use them commercially. 


\section{Introduction}

Familial hypercholesterolemia $(\mathrm{FH})$ is an inherited genetic condition characterized by elevated low-density lipoprotein (LDL) levels and an associated increased risk of atherosclerosis. The carotid intima-media thickness (IMT) measurement is an established method for indirect atherosclerosis risk assessment. The computed tomography (CT)-based calcium score measurement is another method which directly indicates the severity of atherosclerosis. The IMT is also associated with atherosclerosis-independent processes such as intimal hyperplasia. However, all known risk factors for atherosclerosis accelerate its thickening. There is a well-established correlation between increased IMT and a higher cardiovascular risk of both cerebrovascular and cardiac events $[1,2]$. Nevertheless, current guidelines on the prevention of cardiovascular diseases do not recommend routine IMT measurement [3]. The IMT offers fairly low test repeatability, which is considered its significant methodological disadvantage. Furthermore, different researchers use different IMT measurement techniques, which makes it difficult to compare their findings [4]. The IMT measurement in patients with $\mathrm{FH}$, as a high-risk group, is performed in order to determine the long-term effect of cholesterol-lowering treatments. It is also used for children and young adults in order to identify particular high-risk patients early in life [5-7]. The IMT measurement may be carried out proximally — just below the bulb (pIMT) of the common carotid artery (CCA), or slightly lower, distally, in the area where the lines demarcating the contour of the intima-media complex run parallel (dIMT). The former method has been the predominant approach in many previous studies and clinical trials [8-10]. However, today, the latter method is more often preferred [11]. The results of the measurement performed using both methods in the same group of patients differ significantly from each other and correlate with different risk factors [12]. However, it seems that the measurement taken just below the bulb, which is the usual location of early atherosclerotic plaque, may better reflect the atherosclerotic tendency compared to the distal measurement.

The aim of this study was to compare the pIMT and dIMT in patients with FH confirmed by genetic testing ( $\mathrm{FH}$ group) and patients with severe nonfamilial hypercholesterolemia, with negative results of genetic testing (NFH group) and to determine the correlation of results with traditional atherosclerotic risk factors and CT-based calcium scores.

\section{Methods}

The study group was selected from 156 consecutive patients with suspected $\mathrm{FH}$, with a minimum score of 3 on the Dutch Lipid Clinic Network diagnostic criteria, and a positive result of genetic testing for $\mathrm{FH}$. The inclusion and exclusion criteria have been previously reported elsewhere [13]. Additionally, 3 patients with known p.(Gly20Arg) gene polymorphism, which is currently considered a polymorphic variant likely associated with a milder $\mathrm{FH}$ phenotype, were not included in the IMT analysis. After the exclusion criteria were applied, 86 patients with $\mathrm{FH}$ were enrolled (35 male $[\mathrm{M}], 51$ female $[\mathrm{F}]$, mean age $49.8 \pm 11.6)$. The control group consisted of 50 patients $(23 \mathrm{M}$, $27 \mathrm{~F}$, mean age $51.5 \pm 9.9$ ) diagnosed with severe hypercholesterolemia around the same period and with a negative genetic test result. No participant had a history of previous cardiovascular episodes. All patients had an ultrasound scan of both carotid arteries and IMT measurements were performed. The IMT was measured proximally and distally along the carotid artery, with each measurement covering a $1 \mathrm{~cm}$-long segment [12]. All scans were taken using a GE Vivid E9 ultrasound scanner and 4.5-12 MHz linear probe (GE $11 \mathrm{~L}$ ). The scanning depth was optimised at $3-5 \mathrm{~cm}$. All scans were digitally recorded alongside the electrocardiogram (ECG) reading. The semi-automatic measurement was taken along the $1 \mathrm{~cm}$ distal wall segment at the peak of the ECG R-wave using an EchoPAC Clinical Workstation (GE) with dedicated software. The first measurement was taken where the common carotid artery begins to widen, forming a bulb (pIMT). The second measurement was taken where the lines demarcating the inner and outer contour of the IMT complex begin to run parallel (dIMT, Fig. 1). The mean IMT (meanIMT) for a given segment and the maximum value for the left and right carotid artery (maxIMT) were then computed. All measurements were performed twice and then averaged. In order to estimate the repeatability of IMT measurements, intraand interobserver variability analysis was also performed on 50 patients from the NFH group, with measurements taken independently by an experienced cardiologist and radiologist. All participants also underwent an ECG-gated cardiac CT with calcium score assessment of coronary arteries, aortic valve and aorta in line with the method previously reported elsewhere [13, 14]. The study protocol was approved by the local ethics committee. 


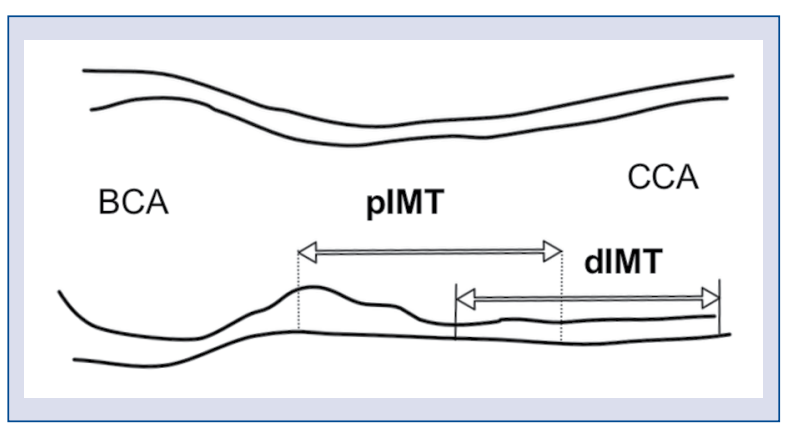

Figure 1. Principles of proximal (pIMT) and distal (dIMT) intima-media thickness measurements. Location determined relative to the common carotid artery (CCA) bulb; $\mathrm{BCA}$ - bulb of the carotid artery.

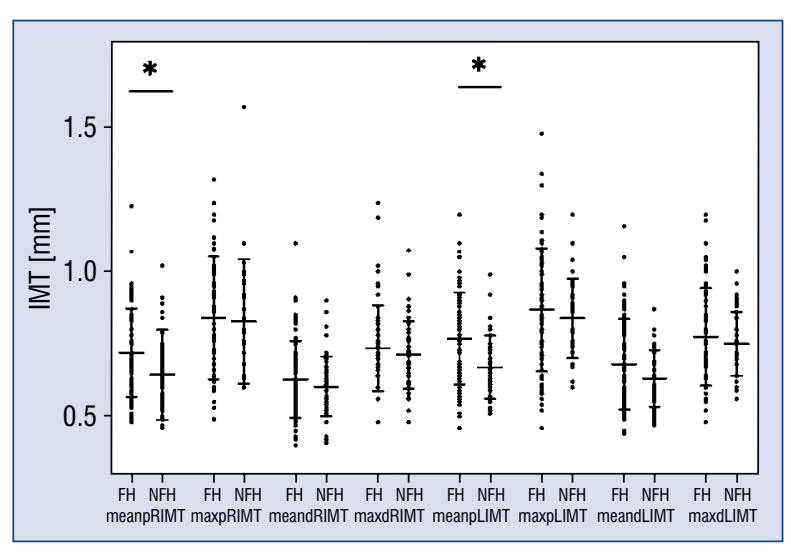

Figure 2. The intima-media thickness of the left and right carotid arteries measured near the bulb (pIMT) and below the bulb (dIMT) in the familial hypercholesterolemia (FH) and non-familial hypercholesterolemia (NFH) groups. ${ }^{*} p<0.01$, there were no significant differences in remaining subgroups; IMT — intima-media thickness; RIMT — right carotid artery IMT; LIMT — left carotid artery IMT; meanpIMT - mean proximal IMT; maxpIMT — maximum proximal IMT; meandIMT mean distal IMT; maxdIMT - maximum distal IMT; pIMT — proximal IMT; dIMT — distal IMT.

\section{Statistical analysis}

The IMT results were presented graphically including mean and standard deviations. The normality of distribution assumption was assessed using the Kolmogorov-Smirnov test. The differences between means were assessed using the student T-test or Mann-Whitney U-test for normally and non-normally distributed variables, respectively. The correlations between the IMT and aortic calcium scores were determined using the Spearman rank correlation coefficient. The associations between IMT and traditional cardiovascular risk factors were determined using the multiple linear regression model. All analyses were carried out using SPSS Statistics software. The results were considered significant for $\mathrm{p}<0.05$.

\section{Results}

The detailed clinical and genetic characteristics of the study group have been previously reported elsewhere [13]. No statistical differences in age, body mass index (BMI), blood pressure, history of diabetes (3\% vs. $10 \%)$, smoking (33\% vs. $42 \%$ ) and pretreatment high-density lipoprotein cholesterol (HDLmax) or triglycerides levels (TG) between the $\mathrm{FH}$ and $\mathrm{NFH}$ groups were found. The $\mathrm{FH}$ group however had higher pretreatment total cholesterol (TCmax, $9.4 \pm 2.2$ vs. $8.1 \pm 1.5, \mathrm{p}<0.001$ ) and low-density lipoprotein cholesterol levels (LDLmax, $7.1 \pm 1.7$ vs. $5.1 \pm 1.1, \mathrm{p}<0.001$ ) The percentage of patients on statin treatment during inclusion to the study also did not significantly differ between the groups ( $53.4 \%$ vs. $40 \%$ ). Figure 2 presents the dIMT and pIMT measurement results. The meanpIMT of both right and left CCA were significantly higher in patients with FH compared to the NFH group. The maxpIMT, meandIMT, and maxdIMT values were higher in the $\mathrm{FH}$ group, although the differences were not significant. The results of multiple linear regression including the IMT parameters and traditional cardiovascular risk factors are shown in Tables 1 and 2. The analysis included the risk factors which did not correlate significantly with each other and correlated with the IMT parameters in a univariate linear regression model. The results of the analysis were presented separately for the right and left carotid artery. Age, pre-treatment cholesterol levels (LDLmax) and systolic blood pressure (SBP) were independent predictors of mean pIMT increase in both carotid arteries. Maximum pIMT values did not correlate significantly with traditional risk factors, except for age. Smoking history, except for the meandLIMT, age and LDLmax were independent predictors of mean and maximum dIMT increase in both carotid arteries. There was no significant correlation between the IMT parameters and diastolic blood pressure, HDLmax and TGmax levels.

The correlations between the IMT parameters and the calcium scores of the aortic valve, ascending aorta, descending aorta and coronary arteries were also evaluated. The results are shown in Table 3. Although it was not high, there was a significant correlation between the calcium scores of the 
Table 1. Results of multivariate regression analysis of the right carotid artery intima-media thickness (IMT) with selected traditional risk factors.

\begin{tabular}{|c|c|c|c|c|c|c|c|c|}
\hline \multirow{3}{*}{$\begin{array}{l}\mathrm{R}^{2} \text { model } \\
\mathrm{P} \\
\text { Variable }\end{array}$} & \multicolumn{2}{|c|}{ meanpRIMT } & \multicolumn{2}{|c|}{ maxpRIMT } & \multicolumn{2}{|c|}{ meandRIMT } & \multicolumn{2}{|c|}{ maxdRIMT } \\
\hline & \multicolumn{2}{|c|}{$\begin{array}{c}0.245 \\
<0.001\end{array}$} & \multicolumn{2}{|c|}{$\begin{array}{l}0.175 \\
<0.01\end{array}$} & \multicolumn{2}{|c|}{$\begin{array}{c}0.306 \\
<0.001\end{array}$} & \multicolumn{2}{|c|}{$\begin{array}{c}0.276 \\
<0.001\end{array}$} \\
\hline & Coeff. & $\mathbf{P}$ & Coeff. & $\mathbf{P}$ & Coeff. & $\mathbf{P}$ & Coeff. & $\mathbf{P}$ \\
\hline Age & 0.004 & $<0.001$ & 0.005 & $<0.01$ & 0.004 & $<0.001$ & 0.004 & $<0.001$ \\
\hline Sex & 0.024 & NS & 0.025 & NS & 0.025 & NS & 0.030 & NS \\
\hline SBP & 0.002 & $<0.05$ & 0.0003 & NS & 0.001 & NS & 0.001 & NS \\
\hline LDLmax & 0.001 & $<0.01$ & 0.001 & 0.07 & 0.0004 & $<0.01$ & 0.0004 & $<0.05$ \\
\hline BMI & 0.001 & NS & 0.09 & 0.07 & 0.005 & 0.08 & 0.005 & 0.09 \\
\hline Smoking & 0.04 & NS & 0.07 & 0.07 & 0.046 & $<0.05$ & 0.062 & 0.01 \\
\hline
\end{tabular}

SBP — systolic blood pressure; LDLmax — maximum value of low-density lipoprotein cholesterol (before treatment); BMI — body mass index; RIMT — right carotid artery IMT; LIMT — left carotid artery IMT; meanpIMT — mean proximal IMT, maxpIMT — maximum proximal IMT; meandIMT - mean distal IMT; maxdIMT — maximum distal IMT; NS — non significant

Table 2. Results of multivariate regression analysis including the left carotid artery intima-media thickness (IMT) and selected traditional risk factors.

\begin{tabular}{|c|c|c|c|c|c|c|c|c|}
\hline & \multicolumn{2}{|c|}{ meanpLIMT } & \multicolumn{2}{|c|}{ maxpLIMT } & \multicolumn{2}{|c|}{ meandLIMT } & \multicolumn{2}{|c|}{ maxdLIMT } \\
\hline $\begin{array}{l}\mathrm{R}^{2} \text { model } \\
\mathrm{P}\end{array}$ & \multicolumn{2}{|c|}{$\begin{array}{c}0.19 \\
<0.01\end{array}$} & \multicolumn{2}{|c|}{$\begin{array}{c}0.07 \\
\text { NS }\end{array}$} & \multicolumn{2}{|c|}{$\begin{array}{c}0.25 \\
<0.001\end{array}$} & \multicolumn{2}{|c|}{$\begin{array}{c}0.255 \\
<0.001\end{array}$} \\
\hline Variable & Coeff. & $\mathbf{P}$ & Coeff. & $\mathbf{P}$ & Coeff. & $\mathbf{P}$ & Coeff. & $\mathbf{P}$ \\
\hline Age & 0.003 & $<0.05$ & 0.003 & NS & 0.004 & $<0.001$ & 0.004 & $<0.01$ \\
\hline Sex & 0.011 & NS & 0.048 & NS & 0.041 & NS & 0.037 & NS \\
\hline SBP & 0.002 & $<0.05$ & 0.0004 & NS & 0.001 & NS & 0.002 & 0.07 \\
\hline LDLmax & 0.001 & $<0.05$ & 0.0004 & NS & 0.001 & $<0.01$ & 0.001 & $<0.05$ \\
\hline BMI & 0.002 & NS & -0.0001 & NS & 0.005 & NS & 0.003 & NS \\
\hline Smoking & 0.047 & NS & 0.041 & NS & 0.026 & NS & 0.058 & $<0.05$ \\
\hline
\end{tabular}

SBP - systolic blood pressure; LDLmax - maximum value of low-density lipoprotein cholesterol (before treatment); BMI — body mass index; RIMT — right carotid artery IMT; LIMT — left carotid artery IMT; meanpIMT — mean proximal IMT, maxpIMT — maximum proximal IMT; meandIMT — mean distal IMT; maxdIMT — maximum distal IMT; NS — non significant

Table 3. Correlation between intima-media thickness (IMT) and calcium scores (Spearman's rank correlation coefficient).

\begin{tabular}{lcccc}
\hline & CCS & TCSasc & TCSdsc & AVCS \\
\hline meanpRIMT & $0.42^{* *}$ & $0.38^{* *}$ & $0.28^{* *}$ & $0.29^{* *}$ \\
maxpRIMT & $0.19^{*}$ & $0.27^{* *}$ & 0.17 & $0.24^{* *}$ \\
meandRIMT & $038^{* *}$ & $0.32^{* *}$ & $0.28^{* *}$ & $0.26^{* *}$ \\
maxdRIMT & $0.36^{* *}$ & $0.30^{* *}$ & $0.27^{* *}$ & $0.23^{* *}$ \\
meanpLIMT & 0.35 & $0.39^{* *}$ & $0.28^{* *}$ & $0.30^{* *}$ \\
maxpLIMT & $0.21^{* *}$ & $0.36^{* *}$ & $0.21^{*}$ & $0.22^{*}$ \\
meandLIMT & $0.29^{* *}$ & 0.38 & 0.13 & $0.30^{* *}$ \\
maxdLIMT & $0.33^{* *}$ & 0.34 & $0.19^{*}$ & $0.21^{*}$ \\
\hline
\end{tabular}

${ }^{*} \mathrm{p}<0.05,{ }^{* *} \mathrm{p}<0.01 ; \mathrm{CCS}$ - coronary calcium score; TCasc - ascending aorta calcium score; TCdsc - descending aorta calcium score; AVCS — aortic valve calcium score; RIMT — right carotid artery IMT; LIMT — left carotid artery IMT; meanpIMT — mean proximal IMT; maxpIMT - maximum proximal IMT; meandIMT — mean distal IMT; maxdIMT — maximum distal IMT 
Table 4. Intraobserver and interobserver variability (defined by intra-class coefficient).

\begin{tabular}{lccc} 
& Intraobserver variability & Interobserver variability & P \\
\hline meanpIMT & 96.2 & 92.9 & $<0.001$ \\
maxpIMT & 94.8 & 90.8 & $<0.001$ \\
meandIMT & 94.9 & 91.9 & $<0.001$ \\
maxdIMT & 93 & 87.1 & $<0.001$ \\
\hline
\end{tabular}

IMT — intima-media thickness; meanpIMT — mean proximal IMT; maxpIMT — maximum proximal IMT; meandIMT — mean distal IMT; maxdIMT — maximum distal IMT

ascending aorta, coronary artery and aortic valve and all IMT parameters. There was a significant correlation between the calcium score of the descending aorta and most IMT parameters, except for the maxpRIMT and meandLIMT. The strongest correlation was shown between the calcium scores and the meanpIMT of both carotid arteries. On the other hand, there was a higher correlation between the IMT parameters and the calcium scores of the coronary arteries and ascending aorta, but lower correlation between the IMT and the calcium score of the descending aorta.

In order to estimate the repeatability of IMT measurements, intra- and interobserver variability analysis was also performed on 50 patients from the NFH group. The results are shown in Table 4. There was a high intra- and interobserver agreement for all analyzed variables, and were higher for the meanIMT than the maxIMT.

\section{Discussion}

A host of studies discuss IMT measurements of patients with hypercholesterolemia. The present study focused on two aspects of IMT measurement, which to date have been rarely discussed. The effect of the measurement site on the IMT values in patients with familial and non-familial severe hypercholesterolemia was assessed. The average values of distal IMT measurements obtained in both subgroups in the current study exceeds the $75^{\text {th }}$ percentile of the normal range as defined in the literature [15]. This corresponds to an increased cardiovascular risk, even though enrolled participants had no history of previous cardiovascular incidents. Furthermore, half of the participants $(49.6 \%)$ had been treated with statins prior to enrolment. Naturally, statin treatment affected the IMT results. However, the percentage of patients on statins in both groups was comparable.

The meanpIMT values in both carotid arteries were significantly higher in the $\mathrm{FH}$ than in the
$\mathrm{NFH}$ group. Although the values of the remaining IMT parameters were higher in the $\mathrm{FH}$ group, the between-group differences were not significant. The TCmax and LDLmax levels were also higher in the FH group, which explains the differences in the meanpIMT. However, the difference in the dIMT was not significant and can be explained by the fact that dispersion of the meandIMT values were lower than those of the meanpIMT values. As a result, it is more difficult to demonstrate measurement site-related differences between two groups of the same size. It should also be noted that as a result of statin treatment administered to some participants, the total cholesterol year score, which reflects the lifetime cumulative total cholesterol, was only slightly (and borderline significantly) higher in the FH group [13]. In patients with hypercholesterolemia from both $\mathrm{FH}$ and $\mathrm{NFH}$ groups, IMT measured proximally to the bulb (pIMT) was higher than IMT measured distally from the bulb (dIMT). Furthermore, pIMT differed significantly between FH and NFH groups. Willekes et al. [16] also found that IMT increases as the distance shortens between the measurement site and the bulb. Studies of cadavers have shown that atherosclerotic plaque in the bulb precedes the onset of atherosclerotic plaque in the common carotid arteries by about three decades [12]. This can be partly explained by the weaker shear stress near the bulb [17], which facilitates lipid penetration into the vascular endothelium [18]. Thus, pIMT is likely to reflect early stages of atherosclerosis earlier than dIMT in patients with hypercholesterolemia. The present findings of higher IMT in the left carotid artery compared to the right carotid artery has been previously described in several studies [19].

In the current analysis, in most cases, there was a significant correlation between IMT parameters and calcium scores, with higher coefficients seen for pIMT than dIMT. This association between IMT and coronary calcium scores has been previously reported in studies carried out in differ- 
ent populations. Arad et al. [20] found a correlation between IMT, coronary calcium scores and the presence of the most hemodynamically significant coronary angiography-confirmed lesion in patients aged 50-75 with coronary artery disease (CAD). Davis et al. [21] also found a strong correlation between IMT and coronary calcium scores in an asymptomatic group of 182 men and 136 women aged 33-42 years, after adjustment for sex and age. Cohen et al. [22] demonstrated a similar correlation in their sample of 150 patients. In their study, CT calcium scoring and IMT thickness measurement were carried out in $61 \%$ of study participants either as a part of cardiovascular prevention or in order to determine the severity of their atherosclerosis. Interestingly, unlike the present study, the highest correlation was found between the calcium scores and the maximum rather than mean IMT. However, their IMT calculation was based on the IMT values measured in the CCA, the bulb and internal carotid artery. In the current study, a significant, but not high, correlation between IMT and calcium scores of not only the coronary arteries but also the aortic valve and the ascending aorta was found. Additionally, there was a significant correlation between the majority of the calculated IMT parameters and descending aorta calcium score, although the correlation coefficient was the lowest. This finding is in line with the Framingham offspring study by Kathiresan et al. [23], who found low correlations between the presence of atherosclerotic plaque in the carotid and coronary arteries, and the aorta. Some authors emphasize that IMT and calcium scores represent different stages of vascular wall degeneration. Furthermore, the presence of calcifications, especially in older aged patients, may not closely correlate with traditional risk factors, such as cholesterol levels, hypertension, diabetes, obesity or history of smoking, whereas these correlations are shown for IMT. Therefore, IMT measurement is believed to be a more sensitive indicator of early atherosclerotic changes [24] whilst calcium scores reflect locally advanced atherosclerosis [21].

Also under analysis herein, was the correlation between traditional risk factors and IMT parameters measured proximally and distally from the carotid artery bulb. Multiple linear regression analysis showed that age, SPB and LDLmax were independent predictors of the mean IMT increase for measurements taken proximally to the bulb, whereas age was the only independent predictor of the maximum pIMT. It has been emphasized that the maximum IMT, being less repeatable than the mean IMT, may reflect more advanced atherosclerotic stages with focal thickening of plaque or represent a sampling error [11]. When measured distally from the bulb, the mean and maximum IMT were similarly predicted by the same independent traditional factors, including age, LDLmax, and smoking history. The similarities between both distal IMT (meandIMT and maxdIMT) parameters can be explained by the fact that these are measured along an even, parallel segment of the carotid artery, therefore both measurements would not differ significantly, unlike meanpIMT and maxpIMT which were measured along an uneven carotid artery segment, which makes measurements significantly discrepant. Age and LDLmax were independent predictors for all meanIMT parameters. The SBP predicted a higher meanpIMT in the present study, whereas positive smoking history predicted a higher value of most distal IMT measurements. The literature data evaluating the effect of IMT measurement site on its correlation with traditional risk factors for atherosclerosis is significantly limited. Polak et al. [12] measured IMT in randomly chosen Caucasian individuals from the MESA study population and found distal IMT to be lower, but correlated better with cardiovascular risk factors than proximal IMT. In another study, the same authors assessed the IMT measurement site as a predictor of $\mathrm{CAD}$ and its effect on the correlation with traditional risk factors [12]. Measurements were taken in 279 randomly chosen Caucasian individuals without a history of previous cardiovascular incidents from the MESA study population. The dIMT better predicted CAD than pIMT. However, diagnosis was only made in 11 patients during the study and therefore, the robustness of the analysis was significantly affected by the low CAD incidence.

\section{Limitations of the study}

Although an increased IMT is generally considered to reflect early atherosclerotic changes, this consideration may not always be true. The IMT is also a measure of smooth muscle hypertrophy reflecting normal aging and the differentiation of those two processes is limited. The main limitation of this single-center study is the relatively small number of patients. Small numbers might have overfitted the multivariable analysis. Nevertheless, the present analysis met all necessary requirements of multivariate logistic regression. Moreover, many of patients with severe hypercholesterolemia enrolled in the study were treated 
over a long period with statins which could have affected the natural history of IMT increase.

\section{Conclusions}

The IMT measured at the carotid artery bulb is higher than the IMT value measured further from the bulb and better differentiates between patients with $\mathrm{FH}$ and NFH. The association between IMT and traditional cardiovascular risk factors varies between measurement sites, which additionally indicates differences in the mechanism of IMT increase depending on the distance from the bulb. The IMT values correlated with coronary, aortic valve and aortic calcium scores in all patients with hypercholesterolemia regardless of genetic etiology.

\section{Acknowledgements}

This study was supported by the "National Center Diagnosis and Treatment of Familial Hypercholesterolemia" grant (grant no. POIG.01.01.0222-079/09; to the Medical University of Gdansk).

\section{Conflict of interest: None declared}

\section{References}

1. O'Leary DH, Polak JF, Kronmal RA, et al. Carotid-artery intima and media thickness as a risk factor for myocardial infarction and stroke in older adults. Cardiovascular Health Study Collaborative Research Group. N Engl J Med. 1999; 340(1): 14-22, doi: 10.1056/NEJM199901073400103, indexed in Pubmed: 9878640.

2. Chambless LE, Heiss G, Folsom AR, et al. Association of coronary heart disease incidence with carotid arterial wall thickness and major risk factors: the Atherosclerosis Risk in Communities (ARIC) Study, 1987-1993. Am J Epidemiol. 1997; 146(6): 483-494, doi: 10.1093/oxfordjournals.aje.a009302, indexed in Pubmed: 9290509.

3. Piepoli MF, Hoes AW, Brotons C, et al. 2016 European Guidelines on cardiovascular disease prevention in clinical practice: The Sixth Joint Task Force of the European Society of Cardiology and Other Societies on Cardiovascular Disease Prevention in Clinical Practice (constituted by representatives of 10 societies and by invited experts)Developed with the special contribution of the European Association for Cardiovascular Prevention \& Rehabilitation (EACPR). Eur Heart J. 2016; 37(29): 2315-2381, doi: 10.1093/eurheartj/ehw106, indexed in Pubmed: 27222591.

4. Lorenz MW, Markus HS, Bots ML, et al. Prediction of clinical cardiovascular events with carotid intima-media thickness: a systematic review and meta-analysis. Circulation. 2007; 115(4): 459-467, doi: 10.1161/CIRCULATIONAHA.106.628875, indexed in Pubmed: 17242284.

5. Lavrencic A, Kosmina B, Keber I, et al. Carotid intima-media thickness in young patients with familial hypercholesterolaemia. Heart. 1996; 76(4): 321-325, doi: 10.1136/hrt.76.4.321, indexed in Pubmed: 8983678.
6. Kusters D, Wiegman A, Kastelein J, et al. Carotid intima-media thickness in children with familial hypercholesterolemia. Circ Res. 2014; 114(2): 307-310, doi: 10.1161/circresaha.114.301430.

7. Wiegman A, de Groot E, Hutten BA, et al. Arterial intima-media thickness in children heterozygous for familial hypercholesterolaemia. Lancet. 2004; 363(9406): 369-370, doi: 10.1016/S01406736(04)15467-6, indexed in Pubmed: 15070569.

8. Kornet L, Lambregts J, Hoeks AP, et al. Differences in nearwall shear rate in the carotid artery within subjects are associated with different intima-media thicknesses. Arterioscler Thromb Vasc Biol. 1998; 18(12): 1877-1884, doi: 10.1161/01. atv.18.12.1877, indexed in Pubmed: 9848879.

9. Iglesias del Sol A, Bots ML, Grobbee DE, et al. Carotid intimamedia thickness at different sites: relation to incident myocardial infarction; The Rotterdam Study. Eur Heart J. 2002; 23(12): 934-940, doi: 10.1053/euhj.2001.2965, indexed in Pubmed: 12069447.

10. Hollander M, Hak AE, Koudstaal PJ, et al. Comparison between measures of atherosclerosis and risk of stroke: the Rotterdam Study. Stroke. 2003; 34(10): 2367-2372, doi: 10.1161/01. STR.0000091393.32060.0E, indexed in Pubmed: 12958327.

11. Iana Simova,. Intima-media thickness: Appropriate evaluation and proper measurement, described. E-journal of the ESC Council for Cardiology Practice 2015; Vol. 13, No 21.

12. Polak JF, Post WS, Carr JJ, et al. Associations of common carotid intima-media thickness with coronary heart disease risk factors and events vary with distance from the carotid bulb. J Am Soc Echocardiogr. 2014; 27(9): 991-997, doi: 10.1016/j. echo.2014.04.019, indexed in Pubmed: 24944141.

13. Galaska R, Kulawiak-Galaska D, Wegrzyn A, et al. Assessment of Subclinical Atherosclerosis Using Computed Tomography Calcium Scores in Patients with Familial and Nonfamilial Hypercholesterolemia. J Atheroscler Thromb. 2016; 23(5): 588-595, doi: 10.5551/jat.31161, indexed in Pubmed: 26666465.

14. Gałąska R, Kulawiak-Gałąska D, Chmara M, et al. Aortic valve calcium score in hypercholesterolemic patients with and without low-density lipoprotein receptor gene mutation. PLoS One. 2018; 13(12): e0209229, doi: 10.1371/journal.pone.0209229, indexed in Pubmed: 30592719.

15. Stein JH, Korcarz CE, Hurst RT, et al. Use of carotid ultrasound to identify subclinical vascular disease and evaluate cardiovascular disease risk: a consensus statement from the American Society of Echocardiography Carotid Intima-Media Thickness Task Force. Endorsed by the Society for Vascular Medicine. J Am Soc Echocardiogr. 2008; 21(2): 93-111; quiz 189, doi: 10.1016/j. echo.2007.11.011, indexed in Pubmed: 18261694.

16. Willekes C, Brands PJ, Willigers JM, et al. Assessment of local differences in intima-media thickness in the human common carotid artery. J Vasc Res. 1999; 36(3): 222-228, doi: 10.1159/000025645, indexed in Pubmed: 10393508.

17. Kornet L, Lambregts J, Hoeks AP, et al. Differences in nearwall shear rate in the carotid artery within subjects are associated with different intima-media thicknesses. Arterioscler Thromb Vasc Biol. 1998; 18(12): 1877-1884, doi: 10.1161/01. atv.18.12.1877, indexed in Pubmed: 9848879.

18. Nerem RM. Vascular fluid mechanics, the arterial wall, and atherosclerosis. J Biomech Eng. 1992; 114(3): 274-282, doi: 10.1115/1.2891384, indexed in Pubmed: 1522720.

19. Sun $\mathrm{Yu}, \mathrm{Lin} \mathrm{CH}, \mathrm{Lu} \mathrm{CJ}$, et al. Carotid atherosclerosis, intima media thickness and risk factors: an analysis of 1781 asymptomatic 
subjects in Taiwan. Atherosclerosis. 2002; 164(1): 89-94, doi: 10.1016/s0021-9150(02)00017-5.

20. Arad Y, Spadaro LA, Roth M, et al. Correlations between vascular calcification and atherosclerosis: a comparative electron beam CT study of the coronary and carotid arteries. J Comput Assist Tomogr. 1998; 22(2): 207-211, doi: 10.1097/00004728199803000-00008, indexed in Pubmed: 9530380.

21. Davis PH, Dawson JD, Mahoney LT, et al. Increased carotid intimal-medial thickness and coronary calcification are related in young and middle-aged adults. The Muscatine study. Circulation. 1999; 100(8): 838-842, doi: 10.1161/01.cir.100.8.838, indexed in Pubmed: 10458720.

22. Cohen GI, Aboufakher R, Bess R, et al. Relationship between carotid disease on ultrasound and coronary disease on CT angiography. JACC Cardiovasc Imaging. 2013; 6(11): 1160-1167, doi: 10.1016/j.jcmg.2013.06.007, indexed in Pubmed: 24229768.

23. Kathiresan S, Larson MG, Keyes MJ, et al. Assessment by cardiovascular magnetic resonance, electron beam computed tomography, and carotid ultrasonography of the distribution of subclinical atherosclerosis across Framingham risk strata. Am J Cardiol. 2007; 99(3): 310-314, doi: 10.1016/j.amjcard.2006.08.028, indexed in Pubmed: 17261388.

24. Wolski C, Rotkiewicz A, Grzelak P, et al. Comparison of tomographic coronary artery calcification index (calcium score) and ultrasonographic measurement of intima-media complex thickness in diabetic subjects. Pol J Radiol. 2011; 76(4): 15-20, indexed in Pubmed: 22802849. 